\title{
MiR-140 Expression Regulates Cell Proliferation and Targets PD-L1 in NSCLC
}

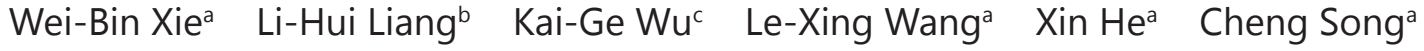 \\ Yun-Qi Wanga Yun-Hui Lid
}

aDepartment of Integrated Traditional Chinese and Western Medicine, Hunan Cancer Hospital and The affiliated Cancer Hospital of Xiangya School of Medicine, Central South University, Changsha, Hunan, bDepartment of Geriatrics, Hunan Provincial People's Hospital, Changsha, Hunan, 'Department of Integrated Traditional Chinese and Western Medicine, Hunan Provincial People's Hospital, Changsha, Hunan, dDepartment of Integrated Traditional Chinese and Western Medicine, Xiangya Hospital, Central South University, Changsha, Hunan, China

\section{Key Words}

Non small cell lung cancer(NSCLC) • PD-L1 • MiR-140 • CyclinE • Cell proliferation

\begin{abstract}
Background/Aims: Programmed death ligand1(PD-L1) plays a role in the development and progression of non-small cell lung cancer (NSCLC). This study aimed to identify miRNA(s) that are responsible for regulation of expression of PD-L1 in NSCLC, and to investigate the role of PD-L1 in regulation of the cell cycle in NSCLC. Methods: We predicted the target miRNA of PD-L1, which was miR-140, using the online tools TargetScan and miBase. In NSCLC cells obtained from clinical specimens, in addition to A549 and NCI-H1650 cell cultures, western blots were used to detect the level of expression of proteins, while real-time PCR was used to determine the level of expression of PD-L1, miR-140, cyclin E, and $\beta$-actin. Transfection with miR-140 mimics, miR-140 inhibitors, and PD-L1 siRNA were conducted using commercial kits. To determine whether miR-140 directly binds PD-L1, a luciferase reporter gene with wild type or mutated PD-L1 was used. Cell viability was measured with the MTT assay, and PI staining was used for cell cycle analysis. Results: We found low expression of miR-140 and high expression of PD-L1 and cyclin E in NSCLC cells. Over-expression of miR-140 suppressed the expression of PD-L1 by directly binding its $3^{\prime}$ UTR, and was also associated with decreased expression of cyclin $\mathrm{E}$ and inhibition of cellular proliferation in A549 and NCI-H1650 cells. Inhibition of PD-L1, in the absence of manipulations to miR-140, also decreased the expression of cyclin E. Conclusion: We conclude that miR-140 directly suppresses PD-L1 and inhibits the miR-140/ PD-L1/cyclin E pathway in NSCLC.

\section{Introduction}

Lung cancer is the leading cause of cancer death worldwide, especially in China [1, 2]. Though multimodal therapies are often used in patients with non-small cell lung cancer (NSCLC), the overall prognosis of NSCLC remains poor [3]. Immunotherapy is reported to be a 
relatively effective strategy for NSCLC treatment. However, clinical treatment of NSCLC using immunotherapy is still limited $[4,5]$ and the underlying mechanisms for immunotherapeutic treatment failures remain largely unknown [6]. Recently, programmed death 1 /programmed cell death ligand-1 (PD-1/PD-L1) has become a popular target for immunotherapeutic blockade of co-inhibitory immune pathways [7]. PD-L1 is the major ligand of PD-1 and is expressed on a variety of cell types [8]. Importantly, PD-L1 is expressed on many tumors, including breast cancer, gastric cancer, NSCLC, pancreaticcancer, bladder cancer, cervical cancer, renal cell carcinoma, and melanoma. Inhibition of the PD-1/PD-L1 pathway enhances antitumor immunity by preventing tumor cells from escaping host immune responses. There is growing evidence that high expression of PD-L1 in NSCLC induces T cell apoptosis and is therefore implicated in escape from immunosurveillance [9]. A growing number of studies have demonstrated that overexpression of PD-L1 promotes proliferation and accelerated carcinogenesis in pancreatic cancer [10]. However, the role of PD-L1 in the proliferation of NSCLC has not yet been elucidated.

miRNAs are small noncoding single-stranded RNA molecules with mature transcripts of 18 to 25 nucleotides long, which are processed from pre-miRNA by the ribonuclease Dicer. miRNAs act as negative regulators of gene expression by binding to the $3^{\prime}$-untranslated region (UTR) of complementary or partially complementary target messenger RNAs (mRNAs), thereby downregulating target mRNAs via degradation or translational inhibition [11]. miRNAs are reported to be involved in both physiological and pathological processes of pantosomatous organs. Multiple miRNAs that may relate to cancer have been discovered, and numerous studies have demonstrated that several miRNAs are up-regulated (miR-21, miR17, miR-221) or down-regulated (miR-15, miR-34a, miR-140) in lung cancer in particular [12-14]. Several studies have reported differential expression of miRNAs in the development of NSCLC [13]. Specifically, it has been reported that miR-140 is down-regulated in NSCLC [15]. However, the role of miR-140 in NSCLC has not been fully investigated, and the target proteins of miR-140 deserve further exploration.

Cyclin E is a key molecule that controls the progression of the cell cycle from $\mathrm{S}$ phase to G2/M phase [16]. Increased cyclin E expression has been consistently associated with shorter survival among stage I to IIIa NSCLC patients [17]. This suggests that cyclin E expression is correlated with the aggressiveness of NSCLC [18-20]. Our bioinformatics analysis, which predicted that PD-L1 and miR-140 are each other's targets, led us to hypothesize that PD-L1 is silenced by miR-140. Furthermore, we hypothesized that expression of PD-L1 is positively related to the expression of cyclin E, and therefore that miR-140 plays a role in regulation of the cell cycle in NSCLC.

In the present study, we measured the expression of miR-140, PD-L1, and cyclin E in clinically-obtained NSCLC cells, and used A549 cells and NCI-H1650 cells to investigate the role of the miR-140/PD-L1/cyclinE pathway in cell proliferation. Our study suggests a crucial role for the miR-140/PD-L1/cyclinE pathway in the pathogenesis of NSCLC, which may suggest a novel mechanism for treatment of NSCLC.

\section{Materials and Methods}

\section{Clinical experiment}

The study was performed following the guidelines set forth in the Declaration of Helsinki. All subjects gave their informed consent, and experiments were approved by the Veterinary Medicine Animal Care and Use Committee of Hunan Cancer Hospital and the affiliated Cancer Hospital of Xiangya School of Medicine. Lung cancer tissue or normal tissue from patients diagnosed with NSCLC was provided by the Laboratory Animal Center, Xiangya School of Medicine, Central South University, China. Before experiments, the tumor tissue and its peripheral normal tissue were frozen in liquid nitrogen.

The tissues were randomly allocated to two groups ( $n=8$ per group): the control group (tissue without cancer) and the NSCLC group (tissue with cancer). The lung tissue was conserved for evaluation of expression of mRNA and protein. 


\section{Cellular Physiology Cell Physiol Biochem 2018;46:654-663 \begin{tabular}{l|l} 
and Biochemistry & $\begin{array}{l}\text { DOI: 10.1159/000488634 } \\
\text { Published on } 2018 \text { The Author(s). Published by S. Karger AG, Basel } \\
\text { www.karger.com/cpb }\end{array}$
\end{tabular} \\ Xie et al.: miR-140 Expression Regulates Cell Proliferation}

Cell culture

NSCLC cell lines A549 and NCI-H1650were purchased from American Type Culture Collection. Cells were cultured with Dulbecco Minimum Essential Medium (DMEM) supplemented with 10\% Fetal Bovine Serum (FBS) in a carbon dioxide incubator at $37^{\circ} \mathrm{C}$ and $5 \% \mathrm{CO}_{2}$. Cells were subcultured and seeded into 6- or 24 -well cell plates for experiments involving transfection. Cells were digested using $0.2 \%$ trypsogen prior to mRNA or protein analysis.

\section{RNA extraction and reverse transcription}

Total RNA was extracted by using TRIzol reagent (TakaRa, Dalian, China). The concentration and purity of RNA was determined spectrophotometrically. Two hundred ng of RNA from each sample was used for the reverse transcription reaction, which was conducted according to the instructions of a transcription kit (DRR037A; TaKaRa, Dalian, China).

\section{Bio-informatics analysis}

We focused on the gene PD-L1, which is up-regulated, and the RNA miR-140, which is down-regulated, in NCSLC tissue. The target gene of miR-140 and the target miRNA of PD-L1 were predicted using the online systems TargetScan and miBase.

\section{Real-time PCR}

Real-time PCR was used to quantitatively analyze the levels of PD-L1 mRNA, cyclin E mRNA, and miR140 in lung tissue or A549 and NCI-H1650cells using SYBR Premix Ex Taq (TaKaRa Dalian, China) with the ABI 7300 real-time PCR system. The real-time PCR primers for the genes PD-L1, cyclin E, and $\beta$-actin are displayed in Table 1. Primers for miR-140 were purchased from RuiBo. Data analysis was performed using the comparative Ct method on ABI software. The result was adjusted by the ratio of PD-L1 or cyclin E to $\beta$-actin, or by the ratio of miR-140 to U6.

\section{Western blotting}

The total protein in each sample was extracted using a protein lysate supplemented with $1 \%$ phenylmethylsulfonyl fluoride (PMSF), which protects proteins from degradation by endogenous protease. Centrifuging at $12000 \mathrm{r} / \mathrm{min}$ produced a supernatant which was used for either denaturation or measuring protein concentration. $40 \mu \mathrm{g}$ protein was used for Western blot analysis by following these steps: Samples were separated on an SDS-PAGE (10\%) gel, then transferred to a polyvinylidene fluoride membrane. The membranes were incubated with rabbit anti-PD-L1 and anti- cyclin E, followed by horseradish peroxidaseconjugated secondary antibodies. To ensure equal loading, blots were also incubated with mouse anti- $\beta$ actin antibodies (Millipore, Billerica, MA, USA). Band signal strength was determined using enhanced chemiluminescence (ECL kit, Amersham Biosciences, Piscataway, NJ, USA) through the Molecular Imager ChemiDoc XRS System (Bio-Rad, Philadelphia, USA).The densitometric analysis was conducted with Image J 1.43 software (National Institutes of Health).

\section{Plasmid transfection}

We constructed two plasmids: wild type(PD-L1-WT) and mutated(PD-L1-MU), each with a luciferase reporter gene. The wild type plasmid contained the seed sequence "UGUGGUA", while the mutated plasmid contained the sequence "UGUAGUA". The two plasmids were amplified in DH5a competent cells and then transfected into A549 and NCI-H1650cellson a 24-well plate at a concentration of $50 \mathrm{ng}$ per well. Meanwhile, A549 cells were co-transfected with miR140 mimics or inhibitors as described below. Finally, cells were collected for analysis of relative luciferase activity.

Transfection with miR-140 mimics

Experiments involving transfection of miR-140

Table 1. The primer sequences were as follows:

\begin{tabular}{lcc}
\hline Primer & & sequenze \\
\hline PD-L1 & F & 5'-ACGCATTTACTGTCACGGTTCC-3' $^{\prime}$ \\
& R & 5'-CGATGGGGTTCCGGCTTCAG-3' $^{\prime}$ \\
cyclin E & F & $5^{\prime}$-ATCCCCACACCTGACAAAGAAG-3' \\
& R & 5'-CCTGAACAAGCTCCATCTGTCA-3' $^{\prime}$ \\
3-actin & F & 5'-ACCGAGCGTGGCTACAGCTTCACC-3' $^{\prime}$ \\
& R & $5^{\prime}$-AGCACCCGTGGCCATCTCTTTCTCG-3'
\end{tabular}
mimics were conducted according to the instructions 
of the manufacturer. Before transfection, a mix including miR-140 mimics and transfection agent lip2000 were prepared. Then, the mix was added into 6- or 24-well plates of A549 orNCI-H1650cell cultures to make a final concentration of $80 \mathrm{nM}$. Transfection proceeded for $6 \mathrm{~h}$, after which the cells were collected for mRNA or protein analysis.

\section{Transfection with PD-L1 siRNA}

PD-L1 siRNA transfection experiments were conducted according to the instructions of a commercial kit. Before transfection, a mix including PD-L1 siRNA and transfection agent EntransterTM-R4000 was prepared. Then, the mix was added into 6- or 24-well plates of A549 orNCI-H1650 cell cultures to make a final concentration of $2 \mathrm{pmol} / \mathrm{L}$. Transfection proceeded for $48 \mathrm{~h}$, after which the cells were collected for mRNA or protein analysis.

\section{MTT staining}

The MTT assay was used according to the manufacturer's instructions to evaluate the proliferation of those A549 cells which were treated with miR-140 mimics. Living cells can reduce exogenous MTT to formazan, which creates a purple sediment in dimethyl sulfoxide (DMSO). The optical density of this sediment, which is proportional to the number of proliferating cells, was measured at $490 \mathrm{~nm}$ using a spectrophotometer.

\section{Cell cycle measurement}

Propidium iodide (PI) staining was used for cell cycle analysis according to the instructions of a commercial kit. A cell suspension was prepared in phosphate buffered saline (PBS), centrifuged at 1500 $\mathrm{rpm}$, fixed with $70 \%$ alcohol at $-20^{\circ} \mathrm{C}$ overnight, centrifuged again, and washed in PBS. PI solution was added to make a final concentration of $50 \mu \mathrm{g} / \mathrm{ml}$, after which the cells were held in the dark for $30 \mathrm{~min}$. We measured distribution of cell phases using flowcytometry, and analyzed the data with Flowjo software.

\section{Statistical analysis}

All assays were repeated independently at least three times. All data were expressed as mean \pm SEM and were analyzed with SPSS 18.0 statistical software.Differences in measured values among the multiple groups were analyzed using analysis of variance with Bonferroni's multiple comparison correction. The Chi-square test was used to compare the frequency distributions between groups. Pearson correlation coefficients were used for correlation analysis. Differences were considered significant at $p<0.05$.

\section{Results}

miR-140 expression is decreased in NSCLC

To begin investigating the role of miR-140 in tumor tissue, we measured the levels of expression of miR-140 in clinically-obtained samples of NSCLC. As shown in Fig. 1A, expression of miR-140, measured with realtime PCR, was significantly decreased in tumor tissues compared with the controls. This suggests that lower miR-140 levels are associated with NSCLC. We examined the relationship between expression of miR-140 and tumor size, and found that expression of miR-140 was negatively correlated with tumor diameter (Fig. 1B). These data suggest that miR-140 plays an important role in NSCLC.

Gene expression of PD-L1 and cyclin E are altered in NSCLC

Bioinformatic analysis using TargetScan and miBase revealed that PD-L1 is a poten-

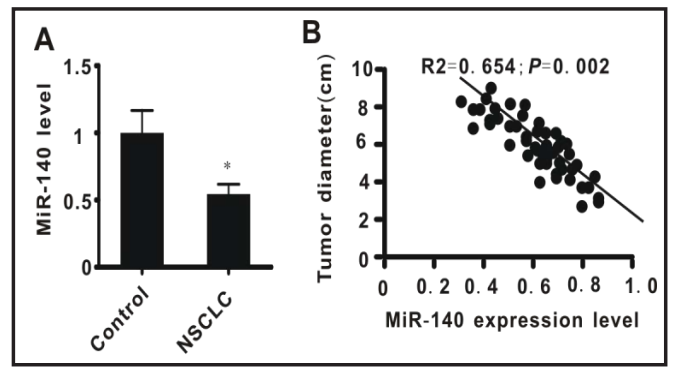

Fig. 1. Expression of miR-140 in NSCLC. miR-140 expression level in lung tissue from each group $(n=42)$; B.Correlation between miR-140 and tumor size. All values are expressed as means \pm SEM. Vs control, ${ }^{*} \mathrm{p}<0.01$. 
Fig. 2. Gene expression in NSCLC. A. Schematic image for the interaction of miR-140 and PD-L1; B. PD-L1 mRNA expression level; C.PD-L1 protein expression level;D. Correlation betweenmiR-140 and PD-L1;E.Cyclin E mRNA expression level; F.Cyclin E protein expression level; G. Correlation betweencyclin E and PD-L1. All values are expressed as means \pm SEM. vs control, ${ }^{*} \mathrm{p}<0.05$.

tial target of miR-140 (Fig. 2A). To investigate the role of PD-L1 and elucidate the relationship between PD-L1 and miR-140 in NSCLC, the levels of expression of PD-L1 and miR-140 were measured using western blot and RTPCR in clinically-obtained NSCLC cells. As shown in Fig. 2, NSCLC was associated with significantly higher expression of PD-L1 compared to control cells. Consistently, analysis revealed an inverse correlation between expression of miR-140 and PDL1 (Fig. 2D). It has been reported that many other genes are altered in NSCLC, including those affecting the cell cycle such as cyclin E. To further investigate the role of cyclin E in NSCLC, we measured the expression of cyclin $\mathrm{E}$ in clinically-obtained NSCLC cells. As shown in Fig. 2E and 2F, the expression of cyclin $\mathrm{E}$ is significantly upregulated in NSCLC cells compared to controls.

Since cell proliferation and apoptosis are two key aspects of tumor progression, we hypothesized that both play a pivotal role in NSCLC. We
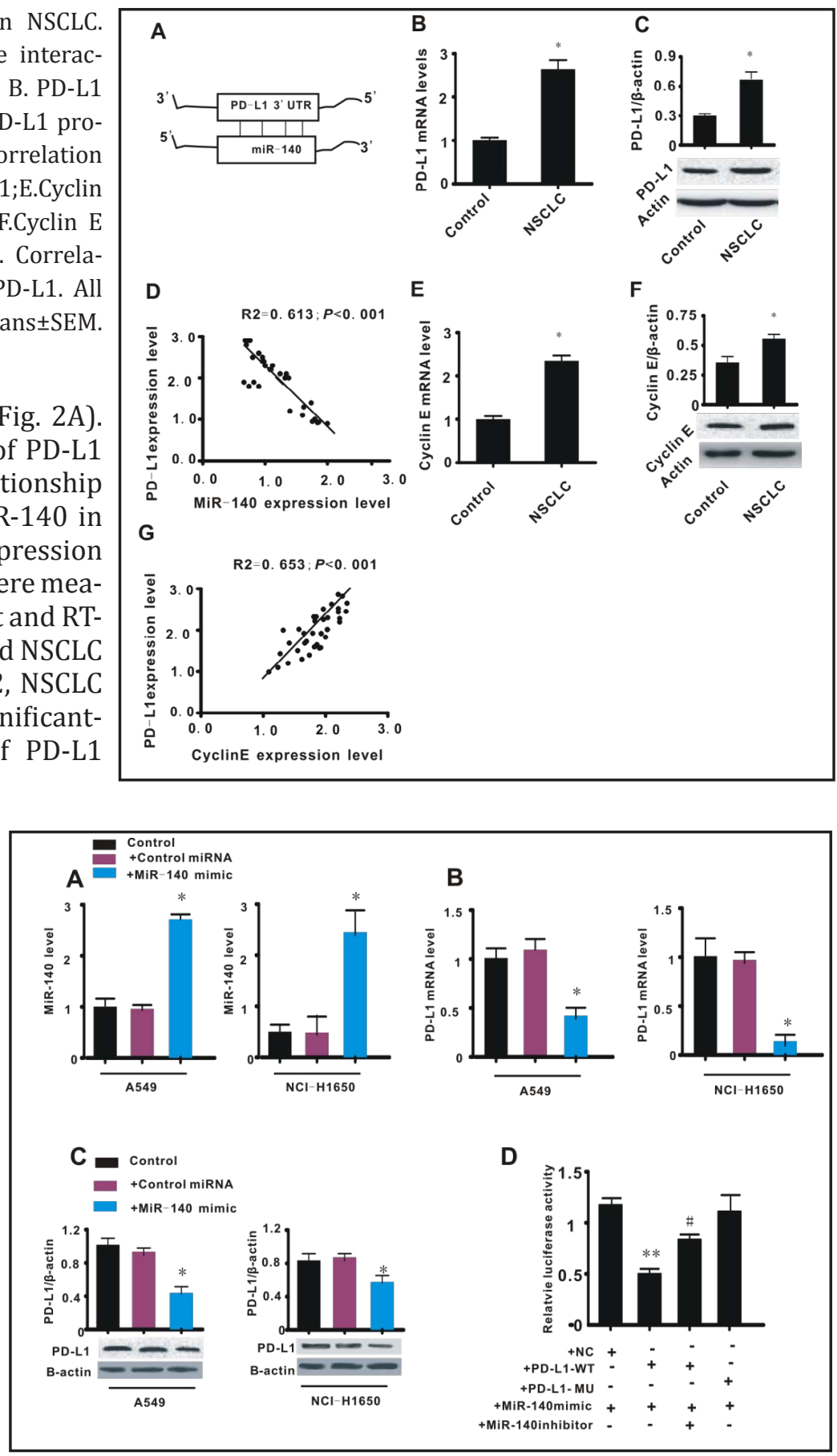

Fig. 3. Effects of miR-140 mimics on the expression of PD-L1 and relative luciferase activity. A. miR-140 expression level; B. PD-L1 mRNA expression level; C. PD-L1 protein expression level; D. Relative luciferase activity. + control miRNA: negative control miRNA; +miR-140 mimics: miR-140 mimics $(50 \mu \mathrm{M})$; +miR-140 inhibitor: miR-140 inhibitor $(100 \mu \mathrm{M})$; +NC: negative control plasmid;+PD-L1-WT: PD-L1-WT plasmid (50ng); +PD-L1-MU: PD-L1-MU plasmid (50ng).All values are expressed as means \pm SEM. vs control, ${ }^{*} \mathrm{p}<0.05$; vs $+\mathrm{NC}$ and +miR140 mimics, ${ }^{* *} \mathrm{p}<0.01$; vs $+\mathrm{NC}$ and + miR140 mimics and +miR140 inhibitor, ${ }^{\mathrm{p}}<0.05$. 
Fig. 4. Effect of miR-140 mimics and PD-L1 inhibition on the expression of cyclin E. A.Cyclin E mRNA expression level; B.Cyclin E protein expression level; C. PD-L1 mRNA expression level; D. PD-L1 protein expression level; E.Cyclin E mRNA expression level; F. Cyclin E protein expression level; +control miRNA: negative control miRNA; +miR-140 mimics: $\operatorname{miR}-140$ mimics $(50 \mu \mathrm{M})$; + control siRNA: negative control SiRNA; +PD-
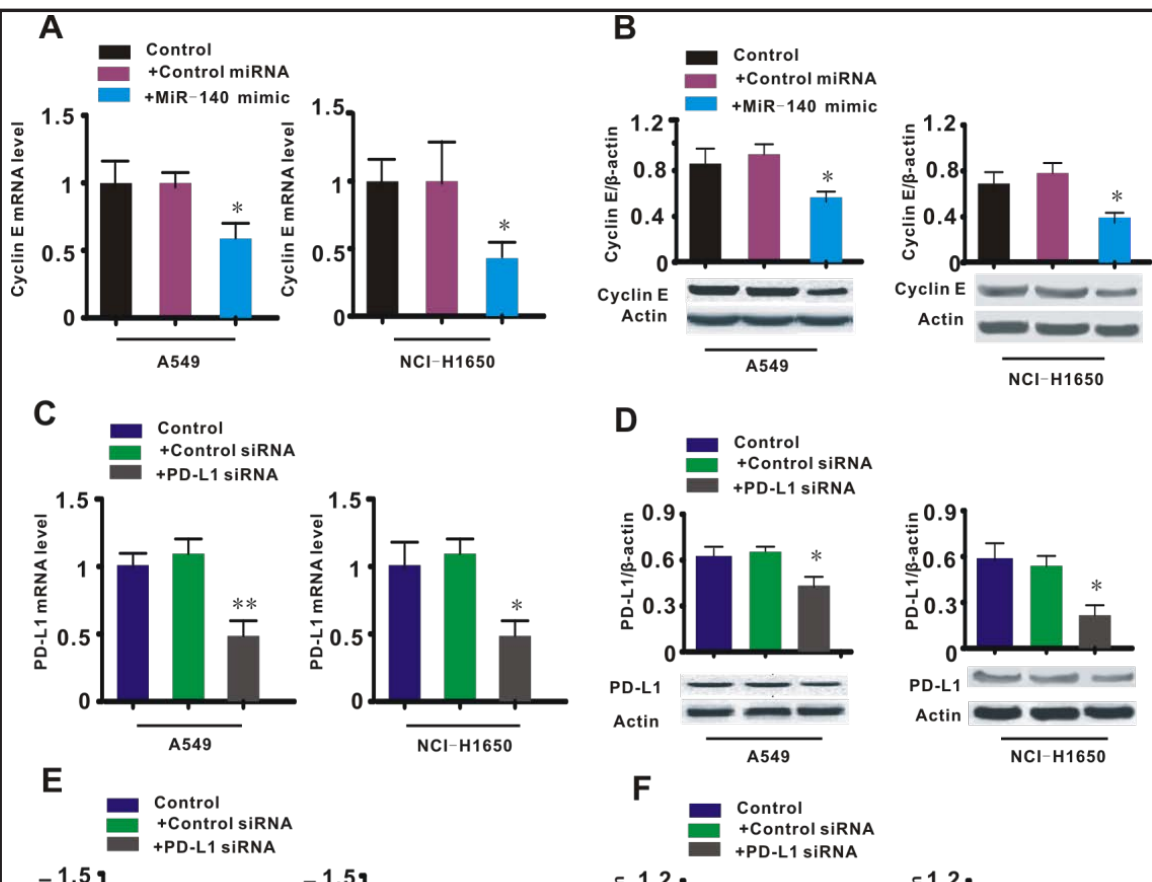

L1siRNA: SiRNA

specific against PD-L1; All values are expressed as means \pm SEM. vs control, ${ }^{*} \mathrm{p}<0.05$.

compared the expression of PD-L1, a gene controlling apoptosis, and the expression of cyclin E, a gene controlling proliferation, and found that they were positively correlated (Fig. 2G). These results indicate that PD-L1 and cyclin E are key players in NSCLC.

\section{miR-140 directly regulates $P D-L 1$ expression}

To confirm the above hypothesis, we co-transfected cells with plasmids containing miR-140 mimics and plasmids containing either wild-type (WT) or mutated (MU) PD-L1/ luciferase reporter genes. As shown in Fig. 3A, the expression of miR-140 was significantly up-regulated in A549 and NCI-H1650 cells after transfection with miR-140 mimics. This suggests that we successfully transfected functional miR-140 mimics into both cell lines. We found that over-expression of miR-140 significantly suppressed PD-L1 expression (both mRNA and protein) (Fig. 3B, C). These results suggest that miR-140 is involved in the regulation of PD-L1 expression. Finally, miR-140 mimics significantly decreased the relative luciferase activity in the PD-L1-WT group, but not in the PD-L1-MU group. In addition, miR140 inhibitors partly reversed the relative decrease in luciferase activity which had been caused by the miR-140 mimics (Fig. 3D). These results indicate that miR-140 suppresses PD-L1 by directly targeting its 3' UTR.

\section{Overexpression of miR-140 or inhibition of PD-L1 decreases cyclin E expression}

Previous studies have found that miR-140 levels are negatively correlated with PD-L1 expression, while PD-L1 expression is positively correlated with cyclin E expression [21, 22]. We therefore hypothesized a relationship between miR-140, PD-L1, and cyclin E. To investigate their roles in expression of cyclin E, the expression of miR-140 and PD-L1were

\section{KARGER}


manipulated. As shown in Fig. 4, miR-140 mimics significantly inhibited expression of cyclin E (Fig. 4A, B). PD-L1-specific siRNA was used to investigate the effect of PD-L1 on cyclin E. siRNA specific to PD-L1 significantly decreased the expression of both PD-L1 (Fig. 4C, D) and cyclin $\mathrm{E}$ (Fig. $4 \mathrm{E}, \mathrm{F}$ ). These results suggest that PD-L1 and miR-140 are involved in the regulation of cyclin E.

$m i R-140$
inhibits the
proliferation
of A549 and
$N C I-H 1650$
cells
To investigate the effects of miR140 on regulation of cellular proliferation in NSCLC, we transfected miR140 mimics into proliferating A549 and NCI-H1650 cells. MTT staining revealed that over-expression of miR-140 significantly decreased the optical density at days 4 and 5 in bothA549 and NCI-

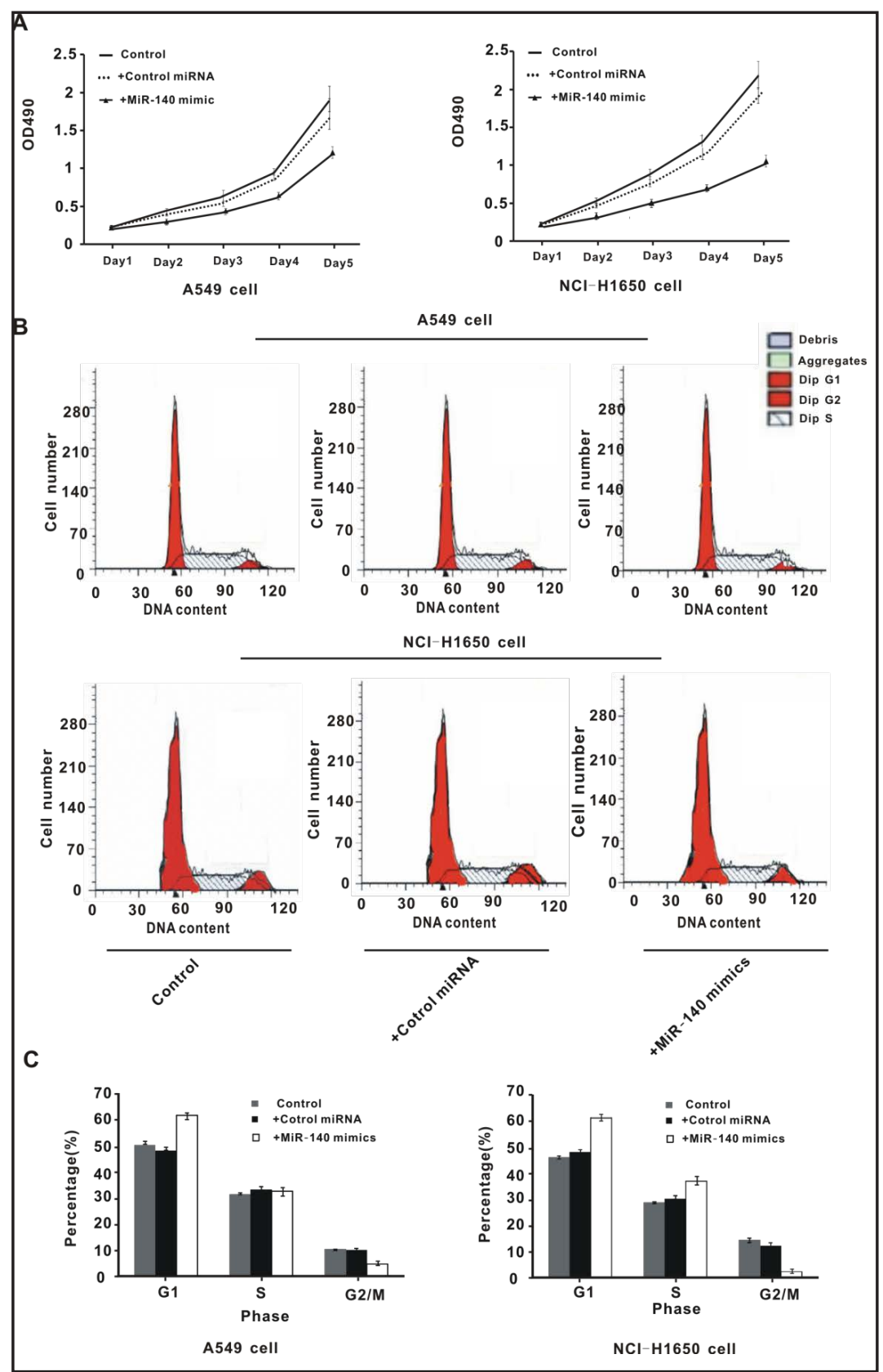

Fig. 5. Effect of miR-140 mimics on the cell cycle of A549 cells and NCI-H1650 cells. A. OD value of MTT measurement from each group; B.Distribution of cell cycles from each group; $\mathrm{C}$. The percentage of cell numbers on the cell cycle. +control miRNA: negative control miRNA; +miR-140 mimics: miR-140 mimics $(50 \mu \mathrm{M})$; All values are expressed as means \pm SEM. ${ }^{*} \mathrm{p}<0.01$ vs control. H1650 cells (Fig. $5 A)$. These results indicate that miR-140 plays an important role in cellular proliferation in NSCLC. To further investigate the effect of miR-140 on the cell cycle, we used PI staining with analysis of the distributions of cell phases. As shown in Fig. 5B and 5C, increased miR-140 contributed to $S$ phase stagnation, without progression to G2/M phase of A549 and NCI-H1650 cells. These results suggest that miR-140 can regulate the cell cycle by inhibiting cellular proliferation. 


\section{Discussion}

In this study, we investigated the roles and mechanisms of miR-140, PD-L1, and cyclin E in NSCLC. We found that NSCLC is associated with low expression of miR-140. Bioinformatics analysis predicted a relationship between miR-140 and PD-L1, we found that miR-140 directly suppresses PD-L1 by interacting with its 3'UTR. Overexpression of miR-140 significantly inhibits the proliferation of A549 and NCI-1650 cells. This may be in part due to its effect on cyclin E expression. These results suggest that the miR-140/PD-L1/cyclinE pathway might be a potential therapeutic target for inhibition of cell proliferation in NSCLC.

Several lines of study have reported that some important, functional miRNAs can be detected in the systemic circulation of patients with NSCLC, these may be very valuable in the diagnosis and prognosis of this disease [23, 24]. Accumulating evidence has shown that miRNAs, including miRNA-377, miR-152, miRNA-126 and miRNA-133b, are involved in the progression and metastasis of NSCLC through various targeted genes includingAEG-1 [25] andneuropilin-1 [26]. These findings suggest that miRNAs might act as novel predictor biomarkers of the progression and metastasis of NSCLC [27].

miR-140 has been intensively used in cancer research, but its role in NSCLC had been poorly understood. In the present study, we found that miR-140 is significantly downregulated in NSCLC, that miR-140 overexpression inhibits NSCLC cell proliferation, and that one target of miR-140 is PD-L1. To corroborate the findings of whether miR-140 expression is involved in tumor growth, migration, and metastasis, we performed the transwell experiment and scratching test. In vitro and in vivo studies have confirmed the antitumor efficacy of PD-1 and PD-L1, which have emerged as important targets for immunotherapy. PD- 1 is a type 1 transmembrane protein of the Ig superfamily $[4,7,8]$, consisting of an extracellular $\mathrm{N}$-terminal IgV-like domain, a transmembrane domain, and a cytoplasmic tail [4]. It engages in inhibitory signal transmission and is expressed on activated immune cell types. PD-1 has two ligands, PD-L1 and PD-L2, which belong to the B7 family. PD-L1 is the major ligand and is expressed on a variety of cell types, importantly including many tumors such as lung cancer. Previous studies have reported that the PD-1/PD-L1 system is involved in tumor immunity and is closely associated with tumorigenesis and invasiveness [28-31]. Increased expression of PD-L1 is significantly associated with poor prognosis in many cancers. Specifically in human NSCLC, it has been associated with tumor aggressiveness and postoperative recurrence [32]. One study demonstrated that increased expression of PDL1 in lung cancer may contribute to escape of tumor cells from the immune response by suppressing maturation of tumor-infiltrating dendritic cells [33]. These interactions may also play a pivotal role in the reduction of specific immune cell apoptosis. Taken together, these data suggest that PD-L1 is involved in tumor infiltration and cell proliferation in NSCLC. Thus, it is reasonable to consider that the blockade of the PD-L1/PD-1 interaction might be a promising anticancer immunotherapy, but controversy still exists regarding the clinical prognostic significance of PD-L1 expression on tumors.

Many studies have indicated consensus agreement that therapeutic blockade of PD-L1 might break multiple layers of immune inhibition, allowing an effective anti-tumor response. Recent studies have demonstrated that PD-L1 is a direct target of HIF1a [34], which suggests that PD-L1 is involved in regulation of cellular proliferation. Indeed, over-expression of PDL1 has been correlated with malignant cell proliferation in pancreatic cancer [35]. These results support the link between PD-L1 and proliferation of cancer cells; in this study, we found that expression of PD-L1 is up-regulated in NSCLC.

Cyclin E is a nuclear protein that is essential for cell cycle progression, DNA replication, and centrosome duplication. Numerous types of cancers are highly associated with dysregulation of cyclin E. Dysregulation of cyclin E occurs in more than $90 \%$ of lung, liver, and gastrointestinal cancers, in addition to bone and breast cancers. Constitutive overexpression of cyclin E induces chromosome instability, impairs normal cell cycle progression, and triggers tumor development in transgenic animal models [16]. Earlier studies have demonstrated that cyclin $\mathrm{E}$ is involved in tumor growth in various cell types, while more 
recent studies have focused on its role in cellular proliferation $[16,36]$. In this study, we showed that expression of cyclin E increased in NSCLC. Furthermore, we found that cyclin E expression was positively correlated with PD-L1 expression,and inhibition of PD-L1 significantly decreased the expression of cyclin E (Fig. 5). This suggests that PD-L1 mediates the regulation of expression of cyclin $\mathrm{E}$.

The present study showed that over-expression of miR-140 resulted in low expression of PD-L1 and cyclin E, led to retardation of A549 and NCI-1650 cell proliferation at S phase, and consequently inhibited tumor proliferation. This study demonstrated that the miR-140/PDL1/cyclin E pathway is involved in the regulation of the cell cycle and cellular proliferation in NSCLC. These findings suggest novel targets for clinical treatment and drug development.

\section{Acknowledgements}

This work was supported by the Hunan Provincial Natural Science Foundation of China (No. 2017JJ3188).

\section{Disclosure Statement}

The authors state no Disclosure Statement.

\section{References}

1 Hong QY, Wu GM, Qian GS, Hu CP, Zhou JY, Chen LA, Li WM, Li SY, Wang K, Wang Q, Zhang XJ, Li J, Gong X, Bai CX: Prevention and management of lung cancer in China. Cancer 2015;121 Suppl 17:3080-3088.

-2 Siegel R, Naishadham D, Jemal A: Cancer statistics, 2012. CA Cancer J Clin 2012;62:10-29.

- Igawa S, Sato Y, Ryuge S, Ichinoe M, Katono K, Hiyoshi Y, Otani S, Nagashio R, Nakashima H, Katagiri M, Sasaki J, Murakumo Y, Satoh Y, Masuda N: Impact of PD-L1 Expression in Patients with Surgically Resected Non-Small-Cell Lung Cancer. Oncology 2017;92:283-290.

4 Chae YK, Pan A, Davis AA, Raparia K, Mohindra NA, Matsangou M, Giles FJ: Biomarkers for PD-1/PD-L1 Blockade Therapy in Non-Small-cell Lung Cancer: Is PD-L1 Expression a Good Marker for Patient Selection? Clin Lung Cancer 2016;17:350-361.

5 Kumar R, Collins D, Dolly S, McDonald F, O'Brien MER, Yap TA: Targeting the PD-1/PD-L1 axis in non-small cell lung cancer. Curr Probl Cancer 2017;41:111-124.

6 Tan M, Song X, Zhang G, Peng A, Li X, Li M, Liu Y, Wang C: Overexpression of adenylate cyclase-associated protein 1 is associated with metastasis of lung cancer. Oncol Rep 2013;30:1639-1644.

-7 Meng X, Liu Y, Zhang J, Teng F, Xing L, Yu J: PD-1/PD-L1 checkpoint blockades in non-small cell lung cancer: New development and challenges. Cancer Lett 2017;405:29-37.

8 Munari E, Zamboni G, Marconi M, Sommaggio M, Brunelli M, Martignoni G, Netto GJ, Moretta F, Mingari MC, Salgarello M, Terzi A, Picece V, Pomari C, Lunardi G, Cavazza A, Rossi G, Moretta L, Bogina G: PD-L1 expression heterogeneity in non-small cell lung cancer: evaluation of small biopsies reliability. Oncotarget 2017;8:90123-90131.

-9 Nishimura H, Nose M, Hiai H, Minato N, Honjo T: Development of lupus-like autoimmune diseases by disruption of the PD-1 gene encoding an ITIM motif-carrying immunoreceptor. Immunity 1999;11:141151.

10 Song X, Liu J, Lu Y, Jin H, Huang D: Overexpression of B7-H1 correlates with malignant cell proliferation in pancreatic cancer. Oncol Rep 2014;31:1191-1198.

11 Martinez B, Peplow PV: Blood microRNAs as potential diagnostic and prognostic markers in cerebral ischemic injury. Neural Regen Res 2016;11:1375-1378.

12 Ricciuti B, Mecca C, Crino L, Baglivo S, Cenci M, Metro G: Non-coding RNAs in lung cancer. Oncoscience 2014;1:674-705.

13 Kong XM, Zhang GH, Huo YK, Zhao XH, Cao DW, Guo SF, Li AM, Zhang XR: MicroRNA-140-3p inhibits proliferation, migration and invasion of lung cancer cells by targeting ATP6AP2. Int J Clin Exp Pathol 2015;8:12845-12852. 


\section{Cellular Physiology Cell Physiol Biochem 2018;46:654-663 \begin{tabular}{l|l} 
and Biochemistry & DOI: 10.1159/000488634 \\
Publisher 2018 The Author(s). Published by S. Karger AG, Basel \\
www.karger.com/cpb
\end{tabular}

14 Green D, Dalmay T, Fraser WD: Role of miR-140 in embryonic bone development and cancer. Clin Sci (Lond) 2015;129:863-873.

15 Gu R, Sun YF, Wu MF, Liu JB, Jiang JL, Wang SH, Wang XL, Guo Q: Biological roles of microRNA-140 in tumor growth, migration, and metastasis of osteosarcoma in vivo and in vitro. Tumour Biol 2016;37:353-360.

16 Cheng PH, Rao XM, Wechman SL, Li XF, McMasters KM, Zhou HS: Oncolytic adenovirus targeting cyclin E overexpression repressed tumor growth in syngeneic immunocompetent mice. BMC Cancer 2015;15:716.

17 Singhal S, Vachani A, Antin-Ozerkis D, Kaiser LR, Albelda SM: Prognostic implications of cell cycle, apoptosis, and angiogenesis biomarkers in non-small cell lung cancer: a review. Clin Cancer Res 2005;11:3974-3986.

18 Huang LN, Wang DS, Chen YQ, Li W, Hu FD, Gong BL, Zhao CL, Jia W: Meta-analysis for cyclin E in lung cancer survival. Clin Chim Acta 2012;413:663-668.

-19 Siu KT, Rosner MR, Minella AC: An integrated view of cyclin E function and regulation. Cell Cycle 2012;11:57-64.

20 Wang G, Sai K, Gong F, Yang Q, Chen F, Lin J: Mutation of isocitrate dehydrogenase 1 induces glioma cell proliferation via nuclear factor-kappaB activation in a hypoxia-inducible factor 1-alpha dependent manner. Mol Med Rep 2014;9:1799-1805.

21 Kapodistrias N, Bobori C, Theocharopoulou G: [MiR-140-3p Downregulation in Association with PDL-1 Overexpression in Many Cancers: A Review from the Literature Using Predictive Bioinformatics Tools]. Adv Exp Med Biol 2017;988:225-233.

22 Ji X, Wang E, Tian F: MicroRNA-140 suppresses osteosarcoma tumor growth by enhancing anti-tumor immune response and blocking mTOR signaling. Biochem Biophys Res Commun 2018;495:1342-1348.

23 Geng Q, Fan T, Zhang B, Wang W, Xu Y, Hu H: Five microRNAs in plasma as novel biomarkers for screening of early-stage non-small cell lung cancer. Respir Res 2014;15:149.

24 Markou A, Sourvinou I, Vorkas PA, Yousef GM, Lianidou E: Clinical evaluation of microRNA expression profiling in non small cell lung cancer. Lung Cancer 2013;81:388-396.

25 Meng F, Zhang L, Shao Y, Ma Q, Lv H: MicroRNA-377 inhibits non-small-cell lung cancer through targeting AEG-1. Int J Clin Exp Pathol 2015;8:13853-13863.

-26 Zhang YJ, Liu XC, Du J: MiR-152 regulates metastases of non-small cell lung cancer cells by targeting neuropilin-1. Int J Clin Exp Pathol 2015;8:14235-14240.

27 Chen SW, Wang TB, Tian YH, Zheng YG: Down-regulation of microRNA-126 and microRNA-133b acts as novel predictor biomarkers in progression and metastasis of non small cell lung cancer. Int J Clin Exp Pathol 2015;8:14983-14988.

-28 Iwai Y, Ishida M, Tanaka Y, Okazaki T, Honjo T, Minato N: Involvement of PD-L1 on tumor cells in the escape from host immune system and tumor immunotherapy by PD-L1 blockade. Proc Natl Acad Sci U S A 2002;99:12293-12297.

29 Qu QX, Xie F, Huang Q, Zhang XG: Membranous and Cytoplasmic Expression of PD-L1 in Ovarian Cancer Cells. Cell Physiol Biochem 2017;43:1893-1906.

-30 Chen L, Xiong Y, Li J, Zheng X, Zhou Q, Turner A, Wu C, Lu B, Jiang J: PD-L1 Expression Promotes Epithelial to Mesenchymal Transition in Human Esophageal Cancer. Cell Physiol Biochem 2017;42:2267-2280.

-31 Li J, Chen L, Xiong Y, Zheng X, Xie Q, Zhou Q, Shi L, Wu C, Jiang J, Wang H: Knockdown of PD-L1 in Human Gastric Cancer Cells Inhibits Tumor Progression and Improves the Cytotoxic Sensitivity to CIK Therapy. Cell Physiol Biochem 2017;41:907-920.

-32 Tang Y, Fang W, Zhang Y, Hong S, Kang S, Yan Y, Chen N, Zhan J, He X, Qin T, Li G, Tang W, Peng P, Zhang L: The association between PD-L1 and EGFR status and the prognostic value of PD-L1 in advanced non-small cell lung cancer patients treated with EGFR-TKIs. Oncotarget 2015;6:14209-14219.

-33 Mu CY, Huang JA, Chen Y, Chen C, Zhang XG: High expression of PD-L1 in lung cancer may contribute to poor prognosis and tumor cells immune escape through suppressing tumor infiltrating dendritic cells maturation. Med Oncol 2011;28:682-688.

-34 Noman MZ, Desantis G, Janji B, Hasmim M, Karray S, Dessen P, Bronte V, Chouaib S: PD-L1 is a novel direct target of HIF-1alpha, and its blockade under hypoxia enhanced MDSC-mediated T cell activation. J Exp Med 2014;211:781-790.

-35 Feng M, Xiong G, Cao Z, Yang G, Zheng S, Song X, You L, Zheng L, Zhang T, Zhao Y: PD-1/PD-L1 and immunotherapy for pancreatic cancer. Cancer Lett 2017;407:57-65.

-36 Kong XY, Zhang DC, Zhuang WX, Hua SH, Dai Y, Yuan YY, Feng LL, Huang Q Teng BG, Yu XY, Liu WZ, Zhang YX: AKAP95 promotes cell cycle progression via interactions with cyclin E and low molecular weight cyclin E. Am J Transl Res 2016;8:811-826. 